\title{
Nanotechnology research directions for societal needs in 2020: summary of international study
}

\author{
Mihail C. Roco • Chad A. Mirkin • \\ Mark C. Hersam
}

Received: 28 January 2011 / Accepted: 30 January 2011/Published online: 17 March 2011

(C) Springer Science+Business Media B.V. (outside the USA) 2011

\begin{abstract}
The paper examines the progress made in nanotechnology development since 2000, achievements at ten years, and opportunities in research, education, innovation and societal outcomes by 2020 worldwide.
\end{abstract}

Keywords Nanoscale science and engineering • Research, education and innovation - Forecast . Governance Societal implications .

International perspective

Nanotechnology is the control and restructuring of matter at the nanoscale, at the atomic and molecular levels in the size range of about $1-100 \mathrm{~nm}$, in order to create materials, devices, and systems with fundamentally new properties and functions because of

This article has been excerpted from: "Nanotechnology Research Directions for Societal Needs in 2020: Retrospective and Outlook", (MC Roco, CA Mirkin, and MC Hersam,), National Science Foundation/World Technology Evaluation Center report, Springer, 2010, Boston (available on http://www.nsf.gov/nano/).

\section{C. Roco ( $\square)$}

National Science Foundation, 4201 Wilson Blvd,

Arlington, VA 22230, USA

e-mail:mroco@nsf.gov

C. A. Mirkin - M. C. Hersam

Northwestern University, Evanston, IL 60208, USA their small structure. The 1999 "Nano1" report Nanotechnology Research Directions: Vision for Nanotechnology in the Next Decade described nanotechnology as a broad-based, multidisciplinary field projected to reach mass use by 2020 and offering a new approach to education, innovation, learning, and governance - a field expected to revolutionize many aspects of human life. ${ }^{1}$ Nanotechnology can profoundly affect the ways the authors live, how healthy the authors are, what the authors produce, how the authors interact and communicate with others, how the authors produce and utilize new forms of energy, and how the authors maintain the environment.

Ten years have passed since that first "Nano1" U.S. National Science and Technology Council report on the prospects for nanotechnology. During this past decade, research and development in nanotechnology has made astonishing progress and has now provided a clearer indication of its potential. This new report ("Nano2") examines the last decade's progress in the field and uncovers the opportunities for nanotechnology development in the United States and around the world in the next decade. It summarizes what has been achieved with the investments made since 2000 ,

\footnotetext{
${ }^{1}$ Roco, M.C., R.S. Williams, and P. Alivisatos, eds. 1999. Nanotechnology research directions: Vision for nanotechnology $R \& D$ in the next decade. Washington, DC: National Science and Technology Council. Also published in 2000 by Springer. Available on http://www.wtec.org/loyola/nano/ IWGN.Research.Directions/
} 
but more importantly, it describes the expected targets for nanotechnology R\&D in the next decade and beyond and how to achieve them in the context of societal needs and other emerging technologies.

The Nano2 report incorporates views of leading experts from academia, industry, and government shared among U.S. representatives and those from over 35 other economies in four forums held between March and July 2010. These began with a brainstorming meeting in Chicago (United States) and included U.S.-multinational workshops in Hamburg, Germany (involving European Union and U.S. representatives); Tokyo, Japan (involving Japan, South Korea, Taiwan, and U.S. representatives); and Singapore (involving Singapore, Australia, China, India, Saudi Arabia, and U.S. representatives). Participants came from a wide range of disciplines, including the physical and biological sciences, engineering, medicine, social sciences, economics, and philosophy.

\section{Outline of the study}

The international study documents the progress made in nanotechnology from 2000 to 2010 and lays out a vision for progress in nanotechnology from 2010 to 2020, in four broad categories of interest:

1. Methods and tools of nanotechnology for investigation, synthesis, and manufacturing

2. Safe and sustainable development of nanotechnology for responsible and effective management of its potential; this includes environmental, health, and safety (EHS) aspects and support for a sustainable environment in terms of energy, water, food, raw materials, and climate

3. Nanotechnology applications for advances in biosystems and medicine; information technology; photonics and plasmonics; catalysis; and high-performance materials, devices, and systems

4. Societal dimensions, including education, investing in physical infrastructure, and governance of nanotechnology for societal benefit

This study is addressed to the academic community, private sector, government agencies, and generally to nanotechnology stakeholders. It aims specifically to provide input for planning of nanotechnology R\&D programs to those producing, using, and governing this emerging field. Significant examples of nanotechnology discoveries and achievements since 2000 and the goals to 2020 are listed in Appendix 1, arranged according to the aforementioned four categories. Five figures illustrate several high-impact applications of nanotechnology (in nanosystems, electronics, biomedicine, catalysts and aeronautics) and U.S. infrastructure investments to support progress in nanotechnology as of 2010.

\section{Progress since 2000}

The broad consensus of forum participants is of strong progress since 2000 in the following areas.

Overall

- The viability and societal importance of nanoscale science, engineering, and technology applications have been confirmed, while extreme predictions, both pro and con, have receded. Advancements in scientific foundation and physical infrastructure were inspired by the 1999 unifying definition and vision of "Nano1."

- Nanotechnology has been recognized as a revolutionary field of science and technology, comparable to the introduction of electricity, biotechnology, and digital information revolutions. Between 2001 and 2008, the numbers of discoveries, inventions, nanotechnology workers, R\&D funding programs, and markets all increased by an average annual rate of 25 percent. The worldwide market for products incorporating nanotechnology reached about $\$ 254$ billion in 2009 (Fig. 1, also see Chapter 13 of the study).

Methods and tools

- New instrumentation has allowed femtosecond measurements with atomic precision in domains of engineering relevance. Single-phonon spectroscopy and sub-nanometer measurements of molecular electron densities have been performed. Single-atom and single-molecule characterization methods have emerged that allow researchers to probe the complex and dynamic 
Fig. 1 Market of final products incorporating nanotechnology: the longterm vision for 2000-2020 (solid line) and outcomes in 2009 (survey by Lux Research, see Chapter 13 of the study). The R\&D focus evolves from fundamental discoveries in 2000-2010 (Nano1 in the figure) to applications-driven fundamental and nanosystem research in 2010-2020 (Nano2)

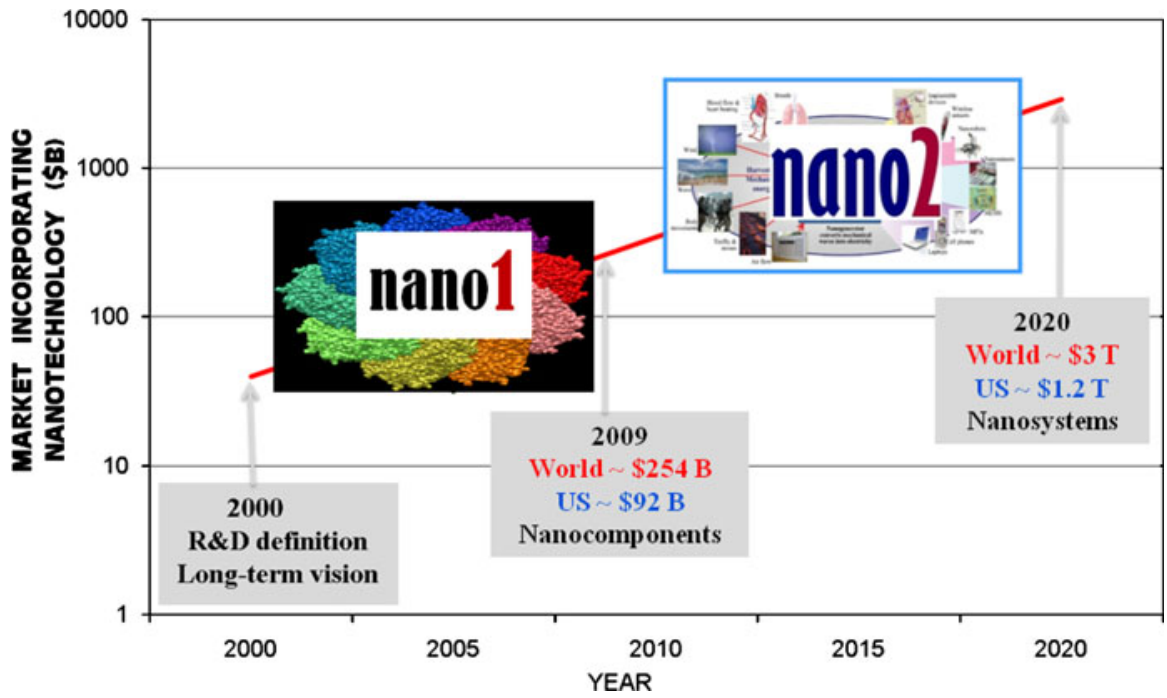

nature of nanostructures in previously impossible ways (Chapter 2). A tool-kit has been established.

- Simulation from basic principles has expanded to assemblies of atoms 100 times larger than in 2000 , and "materials by design" can now be done for a few polymeric and other nanostructures (Chapter 1).

- Fundamental structure-function studies for nanomaterials have led to the discovery and development of important new phenomena such as plasmonics, negative index of refraction in IR/ visible wavelength radiation, Casimir forces, nanofluidics, nanopatterning, teleportation of information between atoms, and biointeractions at the nanoscale. Other nanoscale phenomena are better understood and quantified, such as quantum confinement, polyvalency, and shape anisotropy. Each has become the foundation for new domains in science and engineering.

- An illustration is the discovery of spin torque transfer (the ability to switch the magnetization of nanomagnet using a spin polarized current), which has significant implications for memory, logic, sensors, and nano-oscillators. A new class of devices has been enabled, as exemplified by the worldwide competition to develop spin torque transfer random access memory (STT-RAM), which will be fully commercialized in the next decade.

- Scanning probe tools for printing one molecule or nanostructure high on surfaces over large areas with sub-50 $\mathrm{nm}$ resolution have become reality in research and commercial settings. This has set the stage for developing true "desktop fab" capabilities that allow researchers and companies to rapidly prototype and evaluate nanostructured materials or devices at point of use.

Safe and sustainable development

- There is greater recognition of the importance of nanotechnology-related environmental, health, and safety (EHS) issues for the first generation of nanotechnology products, and of ethical, legal, and social implications (ELSI) issues. Considerable attention is now being paid to building physico-chemical-biological understanding, regulatory challenges for specific nanomaterials, governance methods under conditions of uncertainty and knowledge gaps, risk assessment frameworks, and life cycle analysis based on expert judgment, use of voluntary codes, and incorporation of safety considerations into the design and production stages of new nano-enabled products. Increased attention includes modes of public participation in decision making and overall anticipatory governance with respect to nanotechnology.

- Nanotechnology has provided solutions for about half of the new projects on energy conversion, 
energy storage, and carbon encapsulation in the last decade.

- Entirely new families have been discovered of nanostructured and porous materials with very high surface areas, including metal organic frameworks, covalent organic frameworks, and zeolite imidazolate frameworks, for improved hydrogen storage and $\mathrm{CO}_{2}$ separations.

- A broad range of polymeric and inorganic nanofibers and their composites for environmental separations (membrane for water and air filtration) and catalytic treatment have been synthesized. Nanocomposite membranes, nanosorbents, and redox-active nanoparticles have been developed for water purification, oil spill cleanup, and environmental remediation.

Toward nanotechnology applications

- Many current applications are based upon relatively simple "passive" (steady function) nanostructures used as components to enable or improve products (e.g., nanoparticle-reinforced polymers). However, since 2005, more sophisticated products with "active" nanostructures and devices have been introduced to meet needs not addressed by current technologies (e.g., point-ofcare molecular diagnostic tools and life-saving targeted drug therapeutics).

- Entirely new classes of materials have been discovered and developed, both scientifically and technologically. These include one-dimensional nanowires and quantum dots of various compositions, polyvalent noble metal nanostructures, graphene, metamaterials, nanowire superlattices, and a wide variety of other particle compositions. A periodic table of nanostructures is emerging, with entries defined by particle composition, size, shape, and surface functionality.

- Entirely new concepts have been proved: first quantum device was built and tested in 2010, first artificial cell with synthetic genome was completed, and first hierarchical structures by design have been calculated.

- A versatile library has been invented of new nanostructures and surface patterning methods that are fueling the development of the field. These include commercialized systems such as a large variety of nanoparticles, nanolayers, nanostructured polymers, metals, ceramics, and composites, optical and "dip-pen" nanolithography, nanoimprint lithography, and roll-to-roll processes for manufacturing graphene and other nanosheets. This said, nanotechnology is still in a formative phase from the standpoints of characterization methods, the level of empiricism in synthesis and manufacturing, and the development of complex nanosystems. More fundamental $\mathrm{R} \& \mathrm{D}$ is needed to address these limitations.

- New processes and nanostructures have been formulated using basic principles from quantum and surface sciences to molecular bottom-up assembly, and have been combined with semiempirical, top-down miniaturization methods for integration into products. Nanotechnology has enabled or facilitated novel research in areas such as quantum computing, computing and communication devices (see Fig. 2), nanomedicine, energy conversion and storage, water purification, agriculture and food systems, aspects of synthetic biology, aerospace, geoengineering, and neuromorphic engineering.

- Nanoscale medicine has made significant breakthroughs in the laboratory, advanced rapidly in clinical trials, and made inroads in applications of biocompatible materials, diagnostics, and treatments (see Fig. 3). Advanced therapeutics such as Abraxane are now commercialized and making a significant impact in treating different forms of cancer. The first point-of-care nano-enabled medical diagnostic tools such as the Verigene System are now being used around the world to rapidly diagnose disease. In addition, over 50 cancertargeting drugs based on nanotechnology are in clinical trial in the United States alone. Nanotechnology solutions are enabling companies such as Pacific Biosciences and Illumina to offer products that are on track to meet the $\$ 1000$ genome challenge.

- There has been extensive penetration of nanotechnology into several critical industries. Catalysis by engineered nanostructured materials impacts $30-40 \%$ of the U.S. oil and chemical industries (see Fig. 4) (Chapter 10 in the study); semiconductors with features under $100 \mathrm{~nm}$ constitute over $30 \%$ of that market worldwide and $60 \%$ of the U.S. market (Chapter on Long View); molecular 


\section{nano2}

\section{Nanoelectronic and nanomagnetic components incorporated into common computing and communication devices, in production in $\mathbf{2 0 1 0}$}
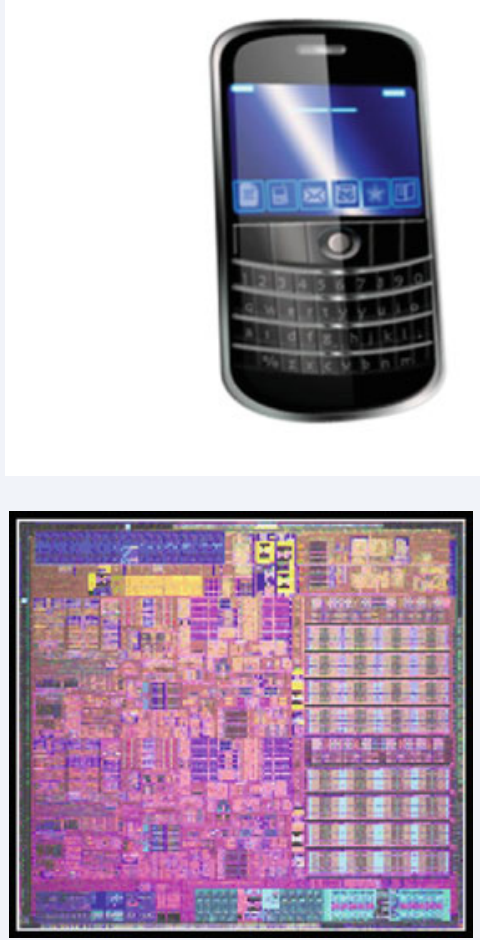

$32 \mathrm{~nm}$ complementary metal oxide semiconductor (CMOS) processor technology by Intel (2009), (gate length of $30 \mathrm{~nm}$ ) with high-K / metal gate. This technology is used to make integrated circuit (IC) chips that will be available in a wide variety of laptop, desktop, and server computer systems, giving higher speed, higher density, and lower power.
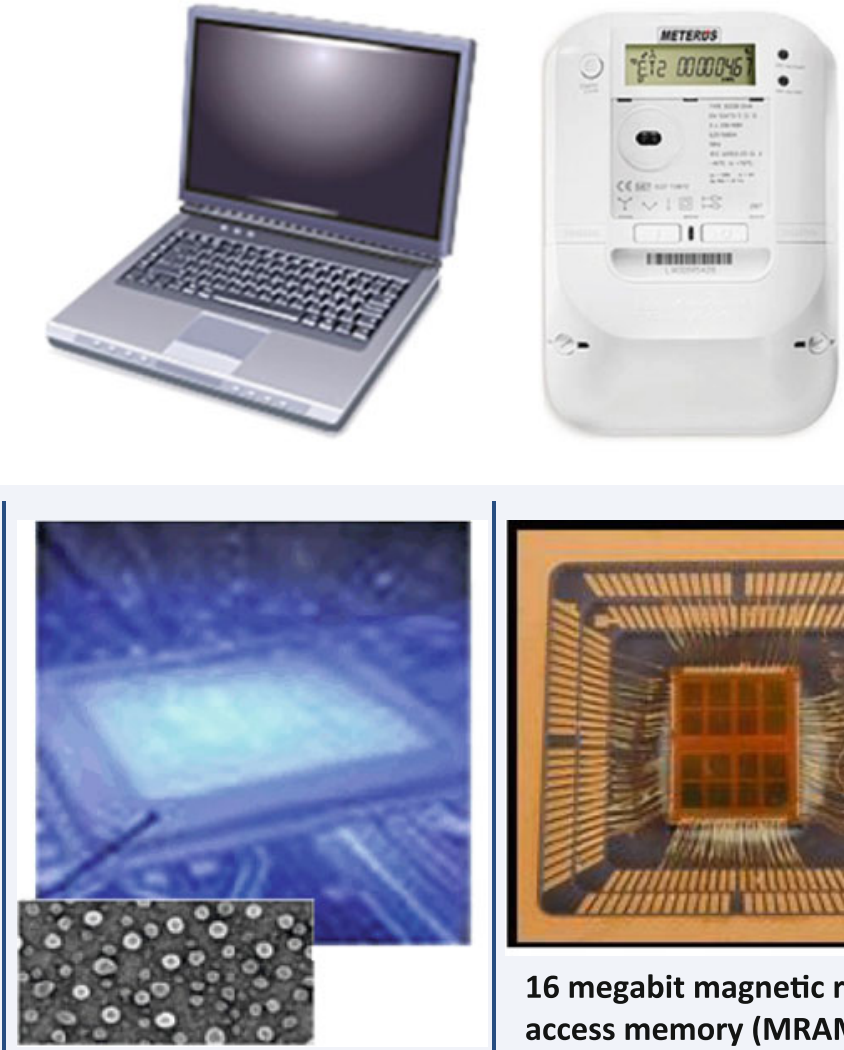

$90 \mathrm{~nm}$ thin-film storage (TFS) flash flexmemory by Freescale (2010) for next-generation microcontrollers, utilizing silicon nanocrystals as the charge storage layer. The nanocrystal layer enables higher-density arrays, lowerpower operation, faster erase times, and improved reliability. Micro-controllers are the "brains" of a wide variety of industrial and consumer products.

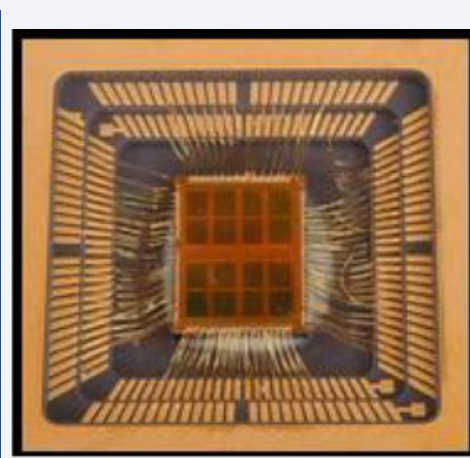

16 megabit magnetic random access memory (MRAM) by Everspin (2010) is based on nanometer-scale magnetic tunnel junctions. These memories have many industrial and commercial applications, such as saving data during a system crash, enabling resume-play features, quick storage and retention of data encryption during shutdown, and retention of vehicle data in an accident for later analysis.

Fig. 2 Illustrations of current nanotechnology applications in cellular phones, computers and medical instrumentation 


\section{nano2}

\section{Examples of nanotechnology incorporated into commercial (and FDA-approved) healthcare products, in production in 2010}

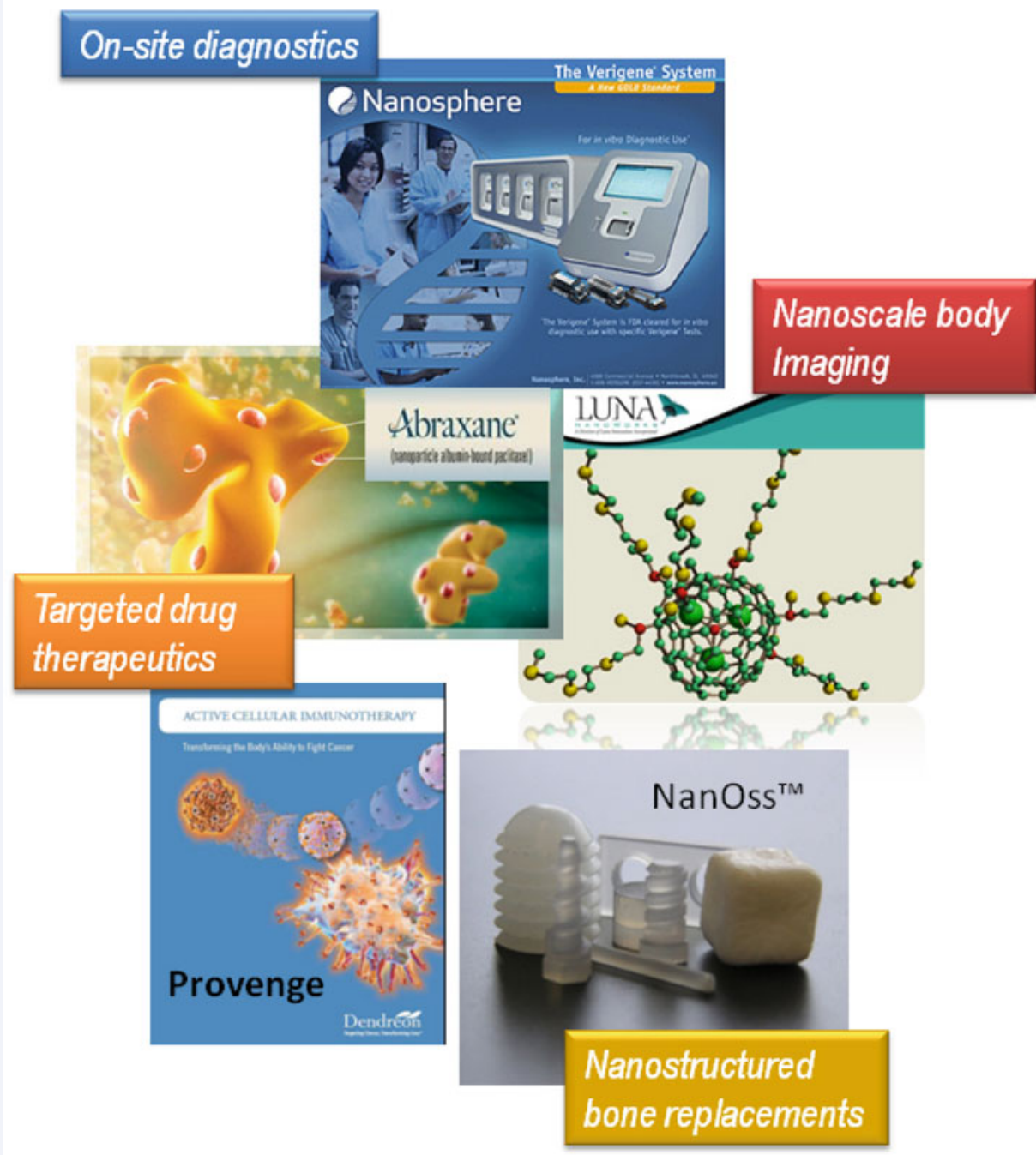

Clockwise from top: Nanosphere Verigene ${ }^{\circledR}$ system for onsite medical diagnostics uses gold nanoparticle technology to detect nucleic acid and protein targets of interest for a wide variety of applications; Luna nanoparticle contrast agents give enhanced clarity and safety of diagnostic magnetic resonance imaging; Angstrom Medica NanOSS ${ }^{\mathrm{TM}}$ nanocrystalline calcium phosphate synthetic bone material is used as bone replacement/reinforcement, weight-bearing bone cement, and bioactive coatings; Dendreon Provenge $₫$ immuno-therapy products are made using cells from patients' own immune systems to fight prostate cancer; and Celgene Abraxane ${ }^{\circledR}$ nanoparticle albumin bound (nab) technology leverages albumin nanoparticles for the active and targeted delivery of chemotherapeutics to treat metastatic breast cancer.

Fig. 3 Illustrations of current nanotechnology applications in nanoscale medicine 


\title{
nano2
}

\section{Examples of nanotechnology in commercial catalysis products for applications in oil refining, 2010}

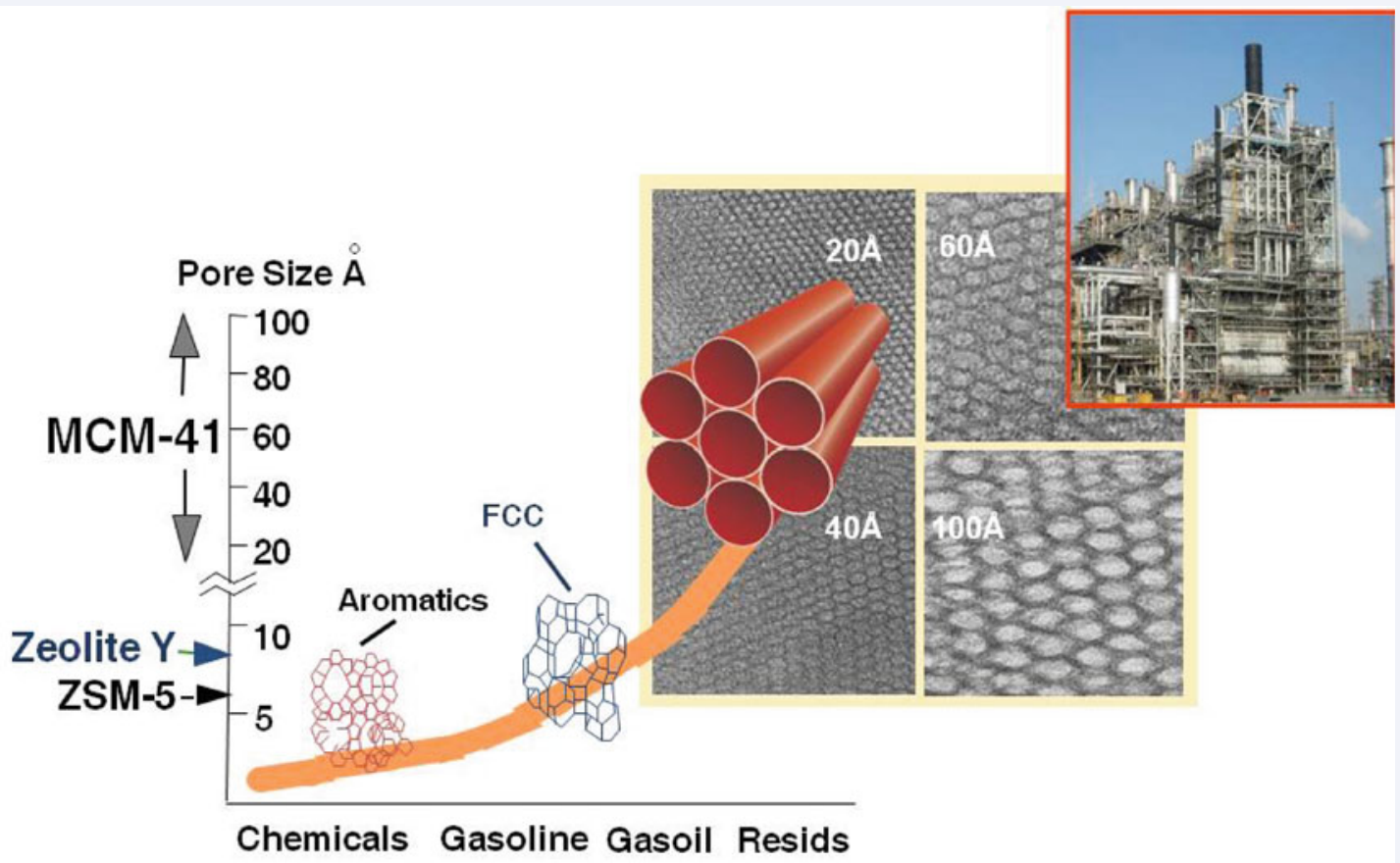

ExxonMobil, Chevron, Dow Chemical, and other oil companies use nanostructured catalysts developed since 2000 for more efficient upgrading of crude oil into transportation fuels and petrochemicals. Redesigned mesoporous silica materials, like MCM-41, along with improved zeolites, are used in a variety of processes such as fluid catalytic cracking (FCC) for producing gasoline from heavy gas oils, and transalkylation for producing para-xylene and related building blocks for the manufacture of polyesters. The global industry upgrades $80+$ million barrels per day (MBD) of petroleum to fuels and chemicals; many of the streams (cuts) are catalytically processed, some multiple times.

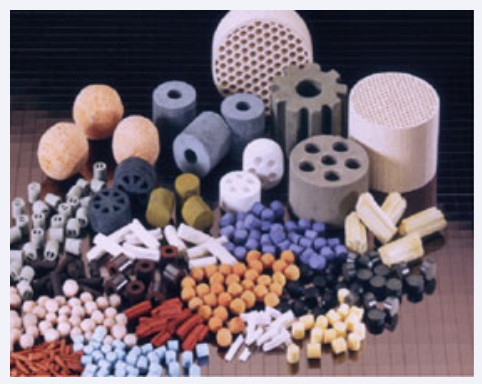

\begin{abstract}
Shape-selective catalysis for managing molecular size and shape is a key application area for modern nanotechnology. The image at left represents many of the nano-engineered catalyst agglomerates of nanoparticles applied to different specialized purposes. Better control of the size, shape, and surface orientation of supported catalyst materials, and better methods to control particulate porosity over multiple length scales, lead to such benefits as increased activity, selectivity, and energy efficiency. In addition, replacement of precious metals by base metal catalysts tailored at the nanoscale is being used to improve productivity and reduce processing costs.
\end{abstract}

Nano-engineered materials now constitute $30-40 \%$ of the huge global catalyst market, which has total annual sales of $\$ 18-20$ billion. The broader, value-added impact of catalytic processing on the U.S. economy alone is estimated at several hundred billion dollars per year.

Fig. 4 Illustrations of current nanotechnology applications in catalysts 
medicine is a growing field. The state of the art in nanoelectronics has progressed rapidly from microscale devices to the realm of $30 \mathrm{~nm}$ and is continuing this trajectory to even smaller feature sizes. These and many other examples show nanotechnology is well on its way to reaching the goal set in 2000 for it to become a "general-purpose technology" with considerable economic impact.

- In the United States, the financial investment in nanotechnology R\&D has been considerable over the last 10 years. The cumulative U.S. Government funding of nanotechnology now exceeds US\$12 billion, placing it among the largest U.S. civilian technology investments since the Apollo Moon-landing program (Nature, Sept. 2010, p. 18). Industry has recognized the importance of nanotechnology and the central role of government in the NNI R\&D. The estimated market for products incorporating nanotechnology is about $\$ 91$ billion in 2009 in the United States (Chapter 13). Finally, approximately 60 countries have adopted nanotechnology research programs, making nanotechnology one of the largest and most competitive research fields globally.

\section{Societal dimensions}

- Various activities have led to establishment of an international community of nanotechnology professionals, a sophisticated R\&D infrastructure, multidisciplinary formal and informal education programs, and diverse manufacturing capabilities spanning the chemical, electronics, advanced materials, and pharmaceutical industries.

- The vision of international collaboration and competition set forth a decade ago, including in multinational organizations, has been realized and has intensified since the first International Dialogue on Responsible Development of Nanotechnology, held in the United States in 2004.

- Nanotechnology has become a model for, and an intellectual focus in, addressing societal implications (ELSI) and governance issues of other emerging new technologies.

- Nanotechnology has catalyzed overall efforts in and attracted talent to science and engineering in the last decade worldwide. Key education networks and research user facilities in the United States in 2010 are illustrated in Fig. 5.

- Nanotechnology has become a model for informal science education of the public on topics of emerging technologies and for building strategic educational partnerships between researcher institutions and public education institutions that benefit the educational goals of both.

\section{Vision for 2020}

Nanotechnology R\&D is expected to accelerate the succession of science and innovation breakthroughs toward nanosystems by design, and to lead to many additional and qualitatively new applications by 2020, guided by societal needs. Nanotechnology will be translated from the research labs to consumer use, motivated by responsiveness to societal challenges such as sustainability; energy generation, conservation, storage, and conversion; and improved healthcare that is lower-cost and more accessible. During the first decade, the main driver was scientific discovery accruing from curiosity-driven research. During the next decade, application-driven research will produce new scientific discoveries and economic optimization leading to new technologies and industries. Such translation will benefit society but will require new approaches in accountable, anticipatory, and participatory governance, and real-time technology assessment. Key points of the consensus vision for nanotechnology $R \& D$ over the next decade are noted below.

Investment policy and expected outcomes

- Major continued investment in basic research in nanotechnology is needed, but additional emphasis in going forward should also be placed on innovation and commercialization, on job creation, and on societal "returns on investment," with measures to insure safety and public participation. With each new generation of nanotechnology products, there is improved focus on economic and societal outcomes.

- The frontiers of nanotechnology research will be transformed in areas such as: 


\section{nano2}

V. U.S. Nanotechnology infrastructure in 2010: (top) Key education networks; (bottom) Research user facilities (see also Research Centers in Appendix D)
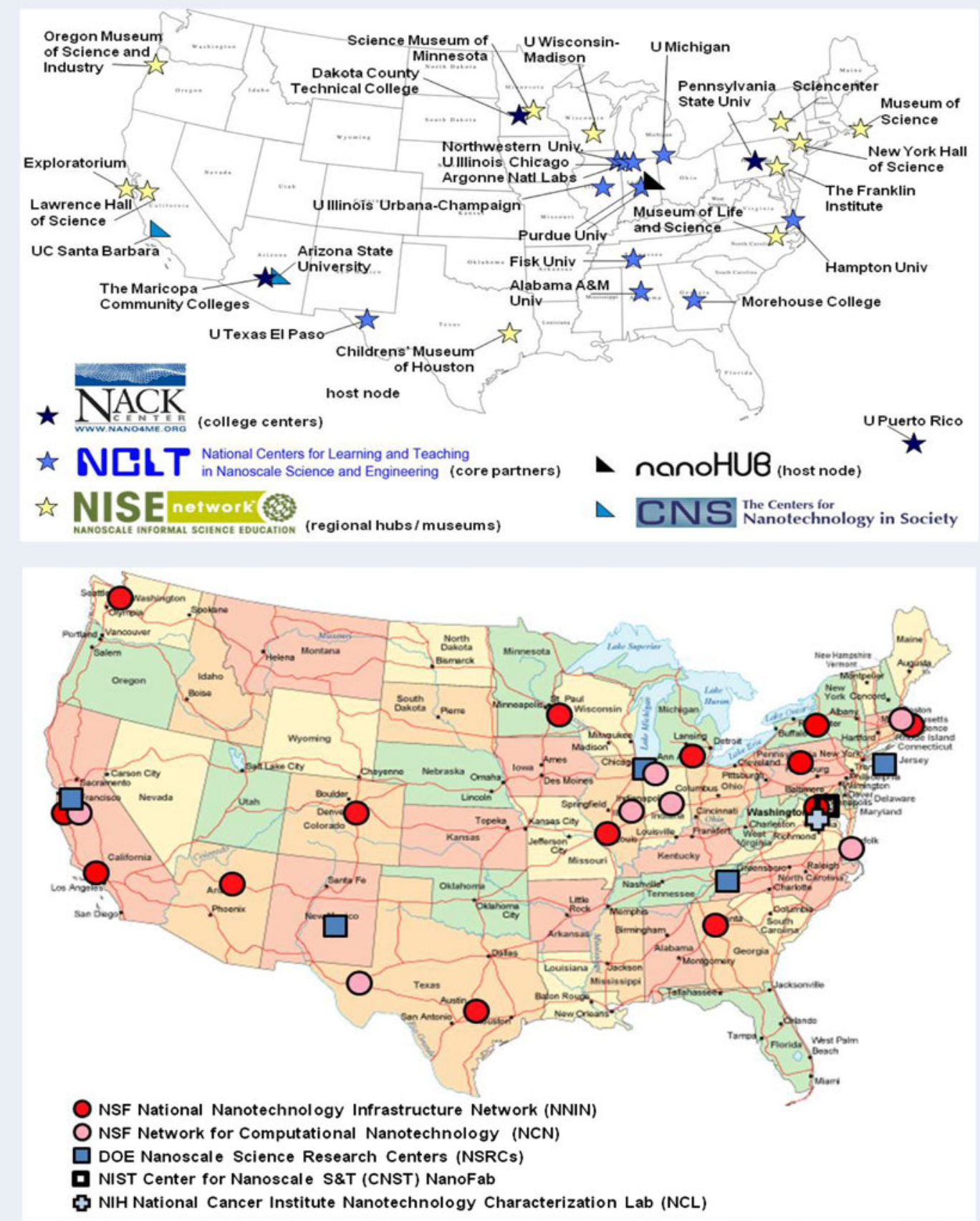

Fig. 5 Illustrations of U.S. nanotechnology infrastructure developed since 2000 
- understanding nanoscale phenomena and processes using direct measurements and simulations

- the classical/quantum physics transition in nanostructures and devices

- multiscale self-assembly of materials from the molecular or nanostructure level upwards

- interaction of nanostructures with external fields

- complex behavior of large nanosystems

- efficient energy harvesting, conversion, and storage with low-cost, benign materials

- understanding of biological processes and of bio-physicochemical interactions at the nanobio interface with abiotic materials

- creation of molecules, materials, and complex systems by design from the nanoscale

- biologically inspired intelligent physical systems for computing

- artificial organs, including the use of fluid networks and nanoscale architectures for tissue regeneration

- personalized instruction for $\mathrm{K}-12$ students on nanotechnology in the form of affordable electronic books that incorporate 3D visual imagery/audio/tactile modes of communication to permit self-paced individualized learning

- direct control and feedback of prosthetics by external sensing of brain activity and/or by direct coupling into the peripheral nervous system associated with the artificial limb

- An innovation ecosystem will be further developed for applications of nanotechnology, including support for multidisciplinary participation, multiple sectors of application, entrepreneurial training, multi-stakeholder-focused research, regional hubs, private-public partnerships, gap funding, and legal and tax incentives.

- Nanotechnology will continue its widespread penetration into the economy as a generalpurpose technology, which-as with prior technologies such as electricity or computing-is likely to have widespread and far-reaching applications across many sectors. For example, nanoelectronics including nanomagnetics has a pathway to devices (including logic transistors and memory devices) with feature sizes below
$10 \mathrm{~nm}$ and is opening doors to a whole host of innovations, including replacing electron charge as the sole information carrier. Many other vital industries will experience evolutionary, incremental nanotechnology-based improvements in combination with revolutionary, breakthrough solutions that drive new product innovations.

- Nanotechnology is expected to be in widespread use by 2020 . There is potential to incorporate nanotechnology-enabled products and services into almost all industrial sectors and medical fields. Resulting benefits will include increased productivity, more sustainable development, and new jobs.

- Nanotechnology governance in research, education, manufacturing, and medicine programs will be institutionalized for optimum societal benefits.

Research methods and tools

- New theories on nanoscale complexity, tools for direct measurements, simulations from basic principles, and system integration at the nanoscale will accelerate discovery.

- Tools for simulation of and physical intervention in cellular processes at the molecular scale will establish scientific bases for health/medical interventions, largely completing the conversion of biology into a quantitative "physico-chemical science" rather than an empirical science.

- In situ characterization tools for operating nanodevices will accelerate innovation in electronics and energy sectors, while in situ probes in realistic environments will enable environmental monitoring, food safety, and civil defense.

- In-depth understanding of principles and methods of nanotechnology will be a condition of competitiveness in sectors such as advanced materials, information technology devices, catalysts, and pharmaceuticals. Development of precompetitive nanoscale science and engineering platforms will provide the foundation for innovation in diverse industry sectors.

Education and physical infrastructure

- Multidisciplinary, horizontal, research-to-application-continuum, and system-application education 
and training will be integrated by the unifying scientific and engineering goals and through new education and training organizations.

- A network of regional hub sites- "Nanotechnology Education Hub Network" - should be established as a sustainable national infrastructure for accelerating nanotechnology education and to implement horizontal, vertical, and integration in educational systems.

- Nanotechnology will enable portable devices that will allow individualized learning anywhere and anytime, as well other modalities of learning using techniques such as brain-machine interaction.

- It will be important to continue to create and maintain centers and hubs as research and user facilities, as well as test beds for development and maturation of innovative nano-enabled device and system concepts. Remote access capabilities will be significantly expanded.

Safe and sustainable development

- A focus on nanotechnology EHS hazards and ELSI concerns must be routinely integrated into mainstream nanotechnology research and production activities to support safer and more equitable progress of existing and future nanotechnology generations.

- Simulations of nanoparticle exposure, bio-distribution, and interaction with biological systems will be integrated in risk assessment frameworks, together with life cycle analysis, use of control standards nanomaterials and expert judgments.

- Application of nanotechnology will significantly lower costs and make economic solar energy conversion costs by about 2015 in the United States, and water desalinization by 2020-2025, depending on the region. Nanotechnology will continue to provide breakthrough solutions for over $50 \%$ of new projects on energy conversion, energy storage, and carbon encapsulation.

- By 2020 nanotechnology will have extended the limit of sustainability in water resources by 10 years. The nanostructured membranes and materials with large surface areas discovered in the last decade will be optimized and scaled-up for a variety of applications, including water filtration and desalination, hydrogen storage, and carbon capture.

Nanotechnology applications

- A library of nanostructures (particles, wire, tubes, sheets, modular assemblies) of various compositions will be developed in industrial-scale quantities.

- New applications expected to emerge in the next decade range from very low-cost, long-life, and high-efficiency photovoltaic devices, to affordable high-performance batteries enabling electric cars, to novel computing systems, cognitive technologies, and radical new approaches to diagnosis and treatment of diseases like cancer.

- As nanotechnology grows in a broader context, it will enable creation or advancements in new areas of research such as synthetic biology, cost-effective carbon capture, quantum information systems, neuromorphic engineering, geoengineering using nanoparticles, and other emerging and converging technologies.

- Nanotechnology developments in the next decade will allow systematic design and manufacturing of nanotechnology products from basic principles, through a move toward simulation-based design strategies that use an increasing amount of fundamental science in applications-driven $\mathrm{R} \& \mathrm{D}$, as defined in the Pasteur quadrant (Stokes 1997, Pasteur's Quadrant: Basic Science and Technological Innovation, Brookings Institution Press).

- Nanotechnology developments will allow increasing the power of computers by about 100,000 times since 2010 and building billion sensor networks by 2020 .

- Nanomedicine will revolutionize the way the authors diagnose and treat people, and in most cases, substantially lower the cost of health care. Personalized and point-of-use diagnostic systems will be used extensively to quickly determine the health of a patient and his or her ability to be treated with specific therapeutics. On the therapeutic side, nanomaterials will be the key to enabling gene therapies for widespread use and an effective means of dealing with antibiotic resistance and the so-called "superbugs." 


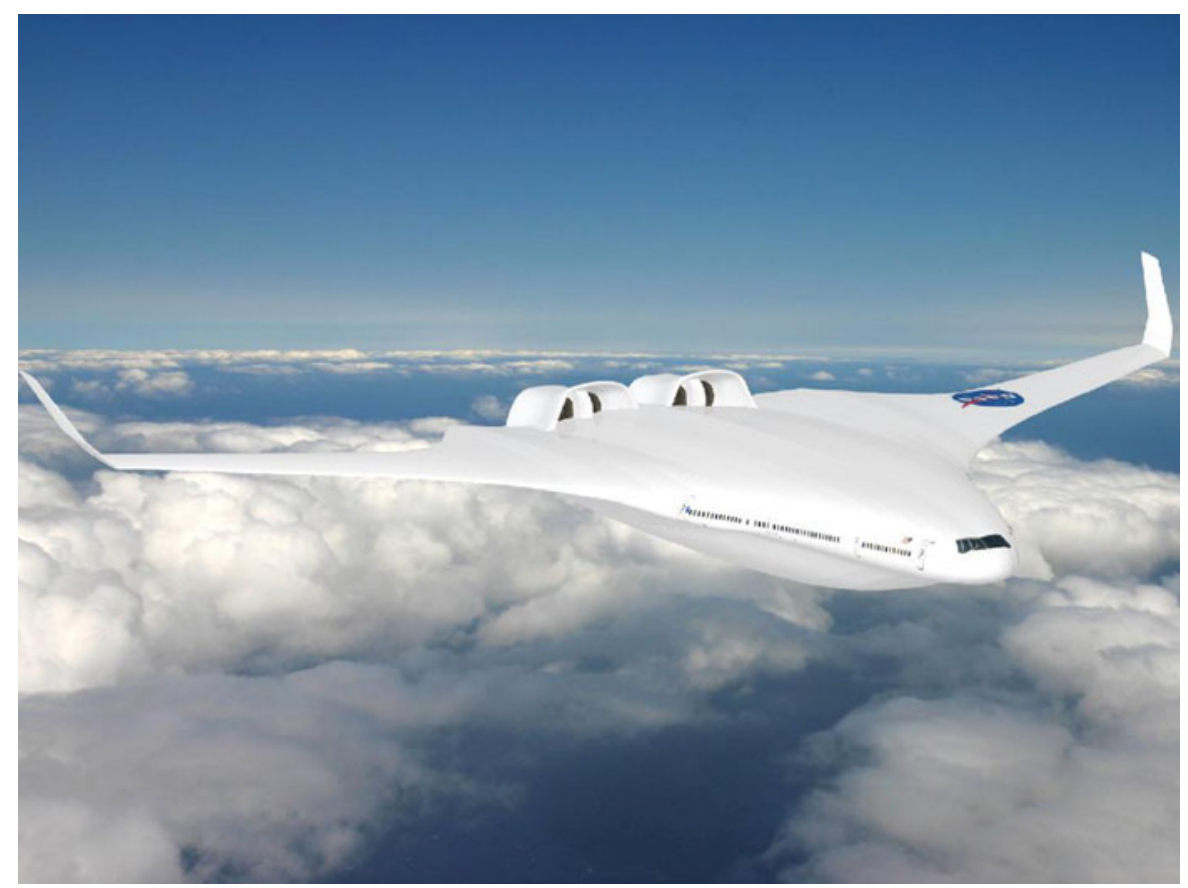

Fig. 6 Design of commercial aircraft integrating several nanotechnology-based solutions (Courtesy of NASA and MIT)

An illustration of nanotechnology application to aeronautics in the future is shown in Fig. 6. This picture suggests the integration of various nanotechnology-based solutions in the design of a blended hybrid-wing-body concept for future subsonic commercial aircraft. It represents a radical departure from conventional subsonic aircraft design. Mechanical and thermal insulation properties of the nanocomposite will allow for "morphing" airframe and propulsion structures that can change their shape or properties on demand to improve aerodynamic efficiency and respond to damage. Composite materials derived from low density, high strength carbon nanotube-based fibers and durable nanoporous matrixes will enable the production of ultra-lightweight multifunctional airframes and with embedded lightning strike protection. Nanotexturing will create surfaces that are naturally resistant to ice accretion thereby eliminating the need for runway deicing and in-flight ice mitigation. Replacement of heavy copper wiring cables with carbon nanotube wires will enable significant reductions in aircraft weight. Distributed autonomous networks of nanotechnology based state sensors powered by high efficiency energy harvesting (thermoelectric, piezoelectric, or photovoltaics) will enable real-time monitoring of the overall health and performance of the aircraft leading to reduced emissions and noise and improved safety. The design, developed by NASA and Massachusetts Institute for Technology, is for a 354 passenger commercial aircraft that would be available for commercial use in 2030-2035 and would enable a reduction in aircraft fuel consumption by $54 \%$ over a Boeing 777 baseline aircraft. (Courtesy of NASA and MIT) (see Fig. 6).

\section{Strategic priorities}

- Continue support for fundamental research, education, and physical infrastructure to change the nanoscale science and engineering frontiers using individual, group, center, and network approaches, with particular focus on direct investigative methods, complex behavior at the nanoscale and how nanoscale behavior controls the microscale/macroscale performance of materials and devices.

- Promote focused $R \& D$ programs, such as "signature initiatives," "grand challenges," and other kinds of dedicated funding programs, to support the development of priority tools, manufacturing capabilities in critical R\&D areas, and a 
nanotechnology-adapted innovation ecosystem. Owing to its pervasiveness, nanotechnology will progressively be integrated with the developments in other emerging and converging technologies.

- Advance partnerships between industry, academia, NGOs, multiple agencies, and international organizations. Give priority to creation of additional regional "nano-hubs" for R\&D, systemoriented academic centers, earlier nanotechnology education, nanomanufacturing, and nanotechnology EHS.

- Support precompetitive $R \& D$ platforms, system application platforms, private-public consortia, and networks in areas such as health, energy, manufacturing, commercialization, sustainability, and nanotechnology EHS and ELSI. The platforms will ensure a "continuing" link between nanoscale fundamental research and applications, across disciplines and sectors.

- Promote global coordination to develop and maintain viable international standards, nomenclatures, databases, and patents and other intellectual property protections. Explore international co-funding mechanisms for these activities. Seek international coordination for nanotechnology EHS activities (such as safety testing and risk assessment and mitigation) and nanotechnology ELSI activities (such as broadening public participation and addressing the gaps between developing and developed countries). An international co-funding mechanism is envisioned for maintaining databases, nomenclature, standards, and patents.

- Develop experimental and predictive methods for exposure and toxicity to multiple nanostructured compounds.

- Support horizontal, vertical, and system integration in nanotechnology education, to create or expand regional centers for learning and research, and to institutionalize nanoscience and nanoengineering educational concepts for K-16 students. Use incentives and competitive methods to harness the energy generated by the students and professors themselves to discover nanotechnology.

- Use nanoinformatics and computational science prediction tools to develop a cross-disciplinary, cross-sector information system for nanotechnology materials, devices, tools, and processes.
- Explore new strategies for mass dissemination, public awareness, and participation related to nanotechnology $\mathrm{R} \& \mathrm{D}$, breaking through gender, income, and ethnicity barriers. This is a great challenge in the next 10 years.

- Institutionalize-create standing organizations and programs to fund and guide nanotechnology activities - in R\&D, education, manufacturing, medicine, EHS, ELSI, and international programs. Important components are the incentivebased, bottom-up programs for research, education, and public participation.

\section{Conclusions}

Several strategic lessons have been learned in the last 10 years:

- There is a need for continued, focused investment in theory, investigation methods, and innovation at the nanoscale to realize nanomaterials and nanosystems by design for revolutionary new products, because nanotechnology is still in a formative phase. Modeling and simulation methods are essential for nanoscale design and manufacturing processes.

- The potential of nanotechnology to support sustainable development in water, energy, minerals, and other resources is higher than realized in the last 10 years; increased R\&D focus is needed.

- Nanotechnology EHS needs to be addressed on an accelerated path as an integral part of the general physico-chemical-biological research program and as a condition of application of the new technology. Knowledge is needed not only for the first generation, but also for the new generation of active nanostructures and nanosystems.

- Besides new emerging areas, more traditional industries may provide opportunities for largescale application of nanotechnology in areas such as mineral processing, plastics, wood and paper, textiles, agriculture, and food systems.

- Multi-stakeholder and public participation in nanotechnology development is essential in order to better address societal dimensions; efforts in this area need to increase. 
- Public-private partnerships need to be extended in research and education.

Nanotechnology is still in an early phase of development, and fundamental understanding and tools are still in the pipeline of new ideas and innovations. Key research themes have been driven by open discovery in the last decade. In the next decade, nanotechnology $R \& D$ is likely to shift its focus to socioeconomic needs-driven governance, with significant consequences for science, investment, and regulatory policies. Likewise, R\&D investment will increasingly focus on science and engineering systems-some with complex and large architectures-that have societal relevance.

It will be vital over the next decade to focus on four distinct aspects of progress in nanotechnology: (1) how nanoscale science and engineering can improve understanding of nature, protect life, generate breakthrough discoveries and innovation, predict matter behavior, and build materials and systems by nanoscale design-knowledge progress; (2) how nanotechnology can generate medical and economic value-material progress; (3) how nanotechnology can promote safety in society, sustainable development, and international collaboration—global progress; (4) how responsible governance of nanotechnology can enhance quality of life and social equity-moral progress.

September 30, 2010

Acknowledgments The NSF/WTEC international study was completed in collaboration with other panel members and expert contributors: Dawn Bonnell, C. Jeffrey Brinker, Mamadou Diallo, Evelyn $\mathrm{Hu}$, Mark Lundstrom, James Murday, André Nel, Mark Tuominen, Jeffrey Welser and Stuart Wolf. The opinions expressed here are those of the authors and do not necessarily represent the position of NSTC/ NSET or NSF.

\section{Appendix 1: Key nanotechnology achievements since 2000 and goals to 2020}

The story of the past decade of nanotechnology R\&D can be described by the developments in fundamental knowledge (e.g., plasmonics), evolutionary or integrative approaches (e.g., integration of nanoelectronics and optoelectronics), and revo- lutionary approaches (e.g., nano-enabled drug delivery using Abraxane) and how these impact each other. Appendix 1 helps to illustrate ways that future achievements can build on past ones

\begin{tabular}{l|l}
\hline Main achievements/discoveries since 2000 & $\begin{array}{l}\text { Fundamental goals/“Holy Grails" to attain } \\
\text { and barriers to overcome by 2020 }\end{array}$ \\
\hline \multicolumn{1}{c}{ Theory, modeling, and simulation } \\
\hline $\begin{array}{l}\text { Discovery of fundamental mechanical, optical, electronic, } \\
\text { magnetic, and biological phenomena, properties, and } \\
\text { processes at the nanoscale }\end{array}$ & $\begin{array}{c}\text { New theories on complexity for concurrent phenomena, and } \\
\text { system integration at the nanoscale that will accelerate } \\
\text { discovery }\end{array}$ \\
$\begin{array}{l}\text { Quantum effects were identified and measured in a series of } \\
\text { nanostructures, such as quantum dots, nanotubes, and } \\
\text { nanowires }\end{array}$ & $\begin{array}{l}\text { Fundamental understanding of the transition from quantum to } \\
\text { classical physics behavior in nanoscale devices and systems } \\
\text { Control and use of quantum phenomena in nanomaterials and } \\
\text { systems }\end{array}$ \\
$\begin{array}{l}\text { Emergence of non-equilibrium green function (NEGF) as a } \\
\text { useful theory of electronic devices }\end{array}$ & $\begin{array}{l}\text { Excited-state electronic structure frameworks including } \\
\text { electronic correlation effects to address 10,000 atoms (and } \\
\text { realistic coupled electron-ion dynamics for 1000). This 100 } \\
\text { advance would open doors to new electron-scale } \\
\text { understanding and potentially to high-throughput evaluation } \\
\text { of new nanomaterials for artificial photosynthesis and other } \\
\text { applications for which energy transport is central }\end{array}$ \\
\hline $\begin{array}{l}\text { Controlled teleportation of information between two atoms in } \\
\text { nanoscale systems }\end{array}$ \\
\hline
\end{tabular}


Appendix 1 continued

Main achievements/discoveries since 2000

Fundamental goals/“Holy Grails" to attain and barriers to overcome by 2020

Advances in atom and nanoparticle level simulations:

- Ab initio, excited-state electronic structure frameworks with realistic treatment of electronic correlation effects

- Molecular dynamic (MD) simulations with chemical bonding

- Self-assembly of functionalized nanoparticles

- Advances in multiscale simulation by coupling of electronic structure theory with MD methods

- Modeling of some nanoparticle-reinforced polymeric composites

Theory of plasmons in metallic nanoparticles and plasmon enhancement of optical processes in molecular and semiconductor systems

Developing understanding of interfaces between biotic and abiotic, natural and manmade, materials, and of nanosystems from the nanoscale

Statistical theories of complex nanostructured materials and devices

10,000 times increase in computational capacity to enable:

- Full Hartree-Foch ab initio simulations of quantum dots

- Simulation of self-assembly of programmed materials

- Automatic generation of force field and reactive force fields for molecular dynamic simulations of materials

- Multiscale whole-cell modeling of tandem solar cells and light-emitting devices

Control and use of plasmonics in nanoscale systems

Predictive approach for compatibility and assembly of biotic and abiotic materials

General approaches to multiscale/multi-phenomena simulation for computational design of nanoscale materials, devices, and integrated nanosystems from basic principles using new models and theories. Simulations will address processes such as self-assembly, catalysis, and dynamics of complex systems

Software packages allowing rapid simulation of optical properties of nanostructures and atomistic-level simulations of nanotransistors

Simulation of quantum transport/current flow at the molecular scale in active nanodevices such as a nanotransistor

\section{Measurement, instrumentation, and standards}

Femtosecond observation of nanoscale interactions (displacement of atoms) in chemical processes

Simultaneous atomic resolution, 3D imaging with chemical specificity and temporal resolution of the nanoscale phenomena. Tools for measuring and restructuring matter with atomic precision, for time resolution of chemical reactions, and for domains of engineering and biological relevance

3D tracking at the single-molecule level of protein motors, enzymes, liposomes, and other bionanostructures

Routine patterning on surfaces becoming scaled up to be useful in applications

3D internal structure imaging with chemical specificity at atomic resolution of an individual protein

Generalized use of reference standard materials and measurement methods in nanoelectronics, biomedical field, nanomanufacturing, and other areas

Measuring probes of continuum properties with atomic resolution, e.g., dielectric function, work function

Develop in situ instrumentation for nanomanufacturing process control

Develop easy-to-use instrumentation for non-specialists and educational applications

Synthesis, assembly, and manufacturing

Creation in the laboratory of a library of nano-components such as particle, tubes, sheets, and 3D structures
Develop a library of nanostructures (particles, wire, tubes, sheets, modular assemblies) of various compositions in industrial-scale quantities 
Appendix 1 continued

Main achievements/discoveries since 2000

Fundamental goals/“Holy Grails" to attain and barriers to overcome by 2020

Creation of relatively simple self-assembled nanostructures

Fundamental understanding of the pathways for self assembly or controllable assembly of atoms or molecules into larger, hierarchical, and stable nanostructures and nanosystems. Better understand the role often played by the presence of a catalytic material or directing structure electrostatic, chemical, and biological interactions) have been tested in laboratory. New polymeric molecules for selfassembling purposes and hierarchical polymeric materials have been obtained by design

Directed assembly using block copolymers, e.g., using grapheo epitaxy for data storage

Creating bio-inspired nanostructures in laboratory conditions (see Chapter 3)

First molecular machines by design were built

Discovery of graphene (in 2004), its unique properties, and its rapid movement toward large-scale production

Introduction of graphene as a viable material or transparent electrodes and large-area roll-to-roll processes for its manufacture

Introduction of graphene as an electronic material, and introduction of processes for its large-area production, to replace indium

Production of chirality-separated carbon nanotubes

Discovery of metamaterials, their unique properties, and their rapid movement toward large-scale production materials-based printing on the nanoscale based upon scanning probe systems (e.g., dip pen, polymer pen, and block copolymer lithographies, etc.)

Contact printing techniques based upon soft elastomers that have become extensively used research tools

Commercialization of high-resolution light-based techniques such as nanoimprint lithography, which are beginning to be used in the semiconductor industry
New concepts of 3D programmable assembly (using

Commercialization of new approaches to molecular and

Systematic approach for design and manufacturing of scalable, hierarchical, directed assembling to three-dimensional structures and devices; programmable assembly

Use both equilibrium and non-equilibrium processes in devices and systems

Expand environmentally benign nanoscale processes

Manufacture nanoproducts that replace hazardous or insufficient materials and existing products

Use existing infrastructure such as lithography and roll-to-roll facilities to create new nanomanufacturing methods

New physics (photons and electron behavior, assembly tools) leading to new applications (faster graphene transistors, $98 \%$ transparent, linking of nanosystems, sensors, composite materials) (Chapter 3)

Sustainably produce carbon nanomaterials integrated into a broad range of electronics products

Use graphene as a foundation for a new generation of planar devices complementing or replacing silicon

Manufacture pure samples of carbon nanotubes to eliminate the need for post-manufacturing sorting

Develop manufacturing methods for nanomaterials

Develop desktop fabs, like desktop printers, that allow researchers to rapidly prototype devices at the point-ofuse at low cost without the requirement of a clean room

Molecular printing techniques that enable single-protein molecule positioning on a surface and exquisite control over important surface-stimulated processes such as stem cell differentiation (at large scale)

Establish full manufacturing tool set and design rules for integration using nanoimprint lithography

Nano-imprinting as a replacement for lithography in some high-resolution applications

\begin{tabular}{l|l}
\hline Realization of video displays using nanotechnology & Mass use of economic, large, and flexible displays \\
\hline $\begin{array}{l}\text { New measurement principles and devices for sensors using } \\
\text { nanoscale structures and phenomena }\end{array}$ & $\begin{array}{c}\text { Develop nanoscale sensor capacity for process monitoring, } \\
\text { health monitoring, and environmental benchmarking and } \\
\text { monitoring }\end{array}$ \\
\hline
\end{tabular}


Appendix 1 continued

Main achievements/discoveries since 2000

Fundamental goals/"Holy Grails" to attain

and barriers to overcome by 2020

\section{Nanotechnology environmental, health, and safety issues}

Development of the concept that the unique properties of engineered nanomaterials allow a wide range of interactions with biomolecules and biological processes that can constitute the basis of nanomaterial hazard as well as be the cornerstone for new diagnosis and treatment options
Further understanding of the nano-bio interface through development of improved instrumentation, rapid-throughput and in silico methodologies that lead to a deeper insight into the biophysicochemical interactions that are required for hazard screening, risk assessment, and safety design of nanomaterials and for improved diagnostics and therapeutics

Demonstration that the use of toxicological injury pathways at the cellular level constitutes a robust scientific basis for knowledge generation about potentially hazardous properties of nanomaterials. The demonstration that oxygen radical production is an important toxicological injury mechanism has resulted in the development of a hierarchical oxidative stress response pathway as the basis for performing hazard ranking of engineered nanomaterials that produce reactive oxygen species biotically and abiotically

Understanding of the importance of developing validated and widely accepted methods for in vitro and in vivo screening of nanomaterial hazards to allow scientists to develop a risk assessment platform commensurate with the growth of nanotechnology

Use of toxicological injury pathways as the basis for highthroughput screening platforms, which would enable largevolume screening, hazard ranking, and prioritization of this information for limited and focused animal experimentation. Although animal experimentation is still necessary to validate the predictiveness of in vitro screening approaches, incremental knowledge generation by smart in vitro procedures could ultimately reduce to a minimum animal experimentation and accompanying costs

Develop predictive toxicological screening methods that allow the correct balance between in vitro and in vivo screening that can be executed by high-throughput technology and in silico decision-making tools that speed up the rate of knowledge generation
Develop experimental and predictive methods for exposure and toxicity to multiple nanostructured compounds and on multiple routes

Establishment of public-private partnerships that have been effective in promoting nano-EHS awareness as well as risk reduction strategies, e.g., the DuPont and Environmental Defense Nano Risk Framework (2007)

Voluntary efforts by leading international scientists to develop harmonized protocols that can be validated by round-robin testing, e.g., the International Alliance of Nano Harmonization

Implementation of the first examples of high-throughput screening assays for nanoparticle hazard assessment

Realization that potential nanoscale hazards must be evaluated as a function of particle size, and that nanomaterials are not necessarily dangerous
Active industry participation in nano-EHS, including in hazard and risk assessment, lifecycle analysis, non-confidential product information disclosure, and implementation of safeby-design strategies

Internationally accepted standards for nanomaterial hazard assessment and risk assessment strategies for the product life cycle nano-informatics and in silico decision-making tools that can help to model and predict nanomaterial hazard, risk assessment, and safe design of nanomaterials
Develop as an integral part of new program development the

\section{Nanotechnology for sustainability: environment, climate, and natural resources}

Awareness of the interdependent, ecosystem-wide implications of human activity on the Earth and the potential of nanotechnology to provide some solutions

Synthesis of a broad range of polymeric and inorganic nanofibers for environmental separations (filtration, membranes) and catalysis

Emergence of electrospinning as a versatile technique for the synthesis of polymeric, inorganic, and hybrid organicinorganic nanofibers
Develop a coordinated approach to use nanotechnology innovation for breakthrough solutions in sustainable development

Solar-powered photocatalytic systems and separation systems (e.g., nanoporous membranes with ion-channel mimics) that extract clean water, energy, and valuable elements (e.g., nutrients and minerals) from impaired water, including wastewater, brackish water, and seawater, with $\sim 99 \%$ water recovery 
Appendix 1 continued

\begin{tabular}{|c|c|}
\hline Main achievements/discoveries since 2000 & $\begin{array}{l}\text { Fundamental goals/"Holy Grails" to attain } \\
\text { and barriers to overcome by } 2020\end{array}$ \\
\hline $\begin{array}{l}\text { Development of nanocomposite membranes (e.g., zeolite } \\
\text { nanocomposite reverse osmosis membranes and } \\
\text { superhydrophobic nanowire membranes), nanosorbents (e.g. } \\
\text { magnetic iron oxide nanoparticles) and redox-active } \\
\text { nanoparticles (e.g., zero valent iron nanoparticles) for water } \\
\text { purification, oil spill clean-up, and environmental } \\
\text { remediation }\end{array}$ & $\begin{array}{l}\text { Integrate functionalized nanofibers and nanoparticles into } \\
\text { systems to develop improved separation and catalytic } \\
\text { systems for } \\
\text { - pollution abatement } \\
\text { - environmental remediation } \\
\text { - green manufacturing }\end{array}$ \\
\hline $\begin{array}{l}\text { Proposals of carbon capture methods using nanotechnology } \\
\text { Discovery of high-porosity nanostructured materials such as } \\
\text { metal organic frameworks (MOFs), covalent organic } \\
\text { frameworks (COFs), and zeolite imidazolate frameworks } \\
\text { (ZIFs) for hydrogen storage and carbon sequestration }\end{array}$ & $\begin{array}{l}\text { Capture carbon and nitrogen using nanostructures and reuse at } \\
\text { industrial scale } \\
\text { Apply multifunctional sorbent/membrane systems with } \\
\text { embedded MOFs, COFs and ZIFs than can selectively extract } \\
\mathrm{CO}_{2} \text { from flue gases and convert it to useful by-products }\end{array}$ \\
\hline $\begin{array}{l}\text { Proposals of geoengineering concepts and experiments using } \\
\text { sulfate or magnetic nanoparticles in upper atmosphere for } \\
\text { sunlight reflection }\end{array}$ & $\begin{array}{l}\text { Develop international projects on geoengineering with control } \\
\text { of the Earth cooling effect and with respect for biodiversity } \\
\text { and environmental safety }\end{array}$ \\
\hline $\begin{array}{l}\text { Methods for efficient use of raw materials using } \\
\text { nanotechnology have been researched }\end{array}$ & $\begin{array}{l}\text { Develop more efficient and environmentally acceptable } \\
\text { separation systems for recovering critical minerals such as } \\
\text { rare earth elements (REE) from mine tailings and wastewater } \\
\text { from mineral/metallurgical extraction and processing plants. } \\
\text { Develop nontoxic, cost-effective REE substitutes and reduce } \\
\text { and (eventually) eliminate the release of toxic pollutants into } \\
\text { soil, water, and air }\end{array}$ \\
\hline
\end{tabular}

\section{Nanotechnology for sustainability: energy}

Rapid improvement in efficiency and scalability of using nanotechnology for solar energy conversion

New solution-processable formulations of inorganic semiconductors for large-area, low-cost photovoltaics

Increased power conversion efficiency of nanostructured organic solar cells by nearly $800 \%$ since 2000
Mass and economic use of nanotechnology for solar energy conversion by $2005-2006$ in United States

Increase module efficiency and decrease production and installation costs to achieve a path toward solar electricity at $\$ 1 / \mathrm{Wp}$ installed

Use of nanoparticles and quantum dots in carrier multiplication and hot-carrier collection strategies to overcome the Shockley-Queisser $31 \%$ efficiency limit in thin-film photovoltaic devices

Improve efficiency of organic photovoltaics from less than $1 \%$ to greater than $8 \%$ through nanoscale phase separation and nanoengineering of device architecture

Improve lifetime of organic photovoltaics through nanotechnology

Replace crystalline silicon with cheap and earth-abundant alternative materials, such as iron disulfide, for photovoltaics

Increased the power density of Li-ion batteries by over $50 \%$, enabling practical hybrid electric vehicles

Nanotechnology-enabled batteries for electric vehicles with large distance range of action

First industrial-scale metal organic frameworks (MOFs) synthesized by BASF in 2010 for hydrogen storage (scale up and deployment of MOFs in gas storage $\left[\mathrm{H}_{2}\right.$ and $\left.\mathrm{CH}_{4}\right]$ )
Increase efficiency of single junction photovoltaic devices to over $31 \%$ through multi-exciton generation, hot carrier harvesting, or other novel phenomena to beat the thermodynamic limit 
Appendix 1 continued

\begin{tabular}{l|l}
\hline Main achievements/discoveries since 2000 & $\begin{array}{l}\text { Fundamental goals/“Holy Grails” to attain } \\
\text { and barriers to overcome by } \mathbf{2 0 2 0}\end{array}$ \\
\hline $\begin{array}{l}\text { Green light emission and other basic discoveries enabling } \\
\text { solid-state lighting }\end{array}$ & $\begin{array}{l}\text { Increase efficiency of solid state lighting to greater than 50\% } \\
\text { through nano-enhanced field intensity (plasmonics) and } \\
\text { emission rate and field coupling capabilities }\end{array}$ \\
\hline \multicolumn{2}{c}{ Nanobiosystems and nanomedicine }
\end{tabular}

Development of diagnostic methods that are sensitive down to picomole and attomole levels and allow for multiple analytes to be assessed simultaneously by lab-on-a-chip approaches
Point of care (POC) medical diagnostics: Many-order-ofmagnitude increased sensitivity, selectivity, and multiplexing capabilities at low cost to enable point-of-care diagnosis and treatment; these capabilities will allow clinicians to track and treat disease - in some cases, years earlier than with conventional tools. Nanodiagnostic tools will become the backbone of clinical medicine by 2020, making the transition from remote labs to hospitals and then eventually to homes

Biological diagnostics: routine live cell imaging with the ability to identify and quantify the key components of a cell (nucleic acids, small molecules, and metal ions) that enable a new way of studying, diagnosing, and treating some of the most debilitating diseases (cancer, cardiovascular disease, and Alzheimer's disease) Nonintrusive diagnostics based on breath and saliva nanoscale detection

Abraxane $^{\circledR}$, the first nanotherapeutic proven to be effective for breast cancer is FDA-approved and a multibillion dollar pharmaceutical; it consists of nanoparticle drug delivery systems, including liposomal, polymer, and albumin nanospheres

Nanotherapeutics: Overcome many challenges such as pharmacokinetics, biodistribution, targeting, tissue penetration, etc., to support widespread adoption by industry of nanotherapeutics

At least $50 \%$ of all drugs used in 2020 will be enabled by nanotechnology; many of these will be for diseases like glioblastoma, pancreatic cancer, and ovarian cancer, where patient prognosis is grim with current therapies

Widespread adoption of nanomaterials by the pharmaceutical community to increase the effectiveness of chemotherapeutics while eliminating toxic side effects

More than 50 of U.S. pharmaceutical companies have nanotechnology-based solutions for treating cancer in clinical testing (Science, Oct. 2010)

Gene therapy enabled by nanomaterials in experimental laboratories; first human trials of siRNA involving a nanomaterial delivery system

Use of temperature-sensitive polymer fibers to coat cell culture dishes for the purposes of cell sheet engineering and demonstrating that the technology can be used for repair of a damaged myocardium, cornea, or esophageal lining (Japan)
$50 \%$ of drugs for pancreatic cancer and ovarian cancer will be nanotechnology-enabled

Clinical approval of gene therapy for treating a wide range of diseases, including many forms of cancer

Inexpensive gene sequencing enabled by nanotechnology

Use nano-architectures and synthetic pro-morphogens for tissue engineering, including stem cell therapy, construction of new organs (e.g., the whole heart or bladder), and spinal cord regeneration

Widespread use by 2020 of nano-enabled tissue constructs for repair of cardiac damage (in heart attack victims) 
Appendix 1 continued

\section{Main achievements/discoveries since 2000}

Fundamental goals/“Holy Grails” to attain

and barriers to overcome by 2020

Controlled development of molecules to promote tissue repair and regeneration in situ
Stem cells: Use nanobiology and nanomedicine to aid in understanding and control of stem cell differentiation and the transition of stem cells to widespread medicinal application; these advances will be fueled by advances in diagnostics, intracellular gene regulation, and high-resolution patterning tools

Multifunctional nanoparticle delivery systems that can be used for drug and siRNA delivery, as well as a combination of both; the multifunctional platform can be further endowed with controllable nano-valves, attachment of surface ligands for cancer tissue, and inclusion or attachment of imaging modalities

Widespread use by 2020 of nanotechnology-enabled stem cellbased therapies for spinal cord regeneration

Achievement of nanoscale control in synthetic biology
Use synthetic biology in regenerative medicine, biotechnology, pharmaceuticals, and energy applications Economic impact: translation of many bionanomaterials to the medical arena, with the market size for these nanomedicine advances growing to $\$ 200$ billion by 2020 , by varying estimates, and in the process dramatically lowering health care costs

\section{Nanoelectronics and nanomagnetics}

Discovery of the quantum spin Hall effect and demonstration of spin transfer torque, which enable direct control of electron spin and magnetic domains by electrical current (see Chapter 8)
Discover multiferroic/magneto-electric materials that will enable spin and magnetic domain control with voltage instead of current

Discover room-temperature collective behavior of carriers in novel materials such as graphene or topological insulators to enable lower-energy nanoelectronic devices

Realize quantum computers for specific uses

First fundamental experiments on quantum computing using small numbers of quantum bits

Continuation of Moore's Law

Scaling of CMOS to $30 \mathrm{~nm}$ dimensions, including an approximate $1 \mathrm{~nm}$ gate insulator, with monolayer accuracy across a $300 \mathrm{~nm}$ wafer

Research, design, and first manufacturing of MRAM nonvolatile memory device

Achieve 3D, near-atomic-level control of reduced-dimensional materials to enable novel nanoelectronic and nanomagnetic behavior

Combine lithography and self-assembly to pattern semiarbitrary structures down to $1 \mathrm{~nm}$ precision

Lower switching current densities and decrease error rate due to thermal fluctuations in the magnetization reversal process in MRAM

Achieve cost-effective architectures of integrated memory and logic using MRAM

Elucidation of electronic, optical, and thermal properties of carbon nanotubes and graphene, and establishment of a new class of electronic materials: carbon electronics

Discover a new logic device capable of switching with energy on the order of a few $\mathrm{kT}$, potentially utilizing an alternative state variable for representing information

Develop a quantum repeater for long-range quantum communication involving teleportation of quantum bits
Discovery of very long spin lifetimes in nitrogen-vacancy (NV) centers in diamond; the quantum state of these centers can be initialized, manipulated, and measured with high fidelity at room temperature 
Appendix 1 continued

Main achievements/discoveries since 2000

Fundamental goals/“Holy Grails" to attain

and barriers to overcome by 2020

\section{Nanophotonics and plasmonics}

Achievement of slowed light in solid state nanophotonic structures (Chapter 10); which enables applications and information systems never before available to photonic systems, such as delaying and storing optical signals

Rapid advances in the field of plasmonics, and the innovations that accompany plasmonics, such as ultra-high resolution optical imaging

First demonstrations of metamaterials (materials with reverse diffraction index) at visible and near-infrared wavelengths

Realization of ultralow-threshold lasers, with thresholds of tens of nanoWatts

\section{Nanostructured catalysts}

Initial ability to characterize some catalytic processes in "the working state"

Control over the size, structure, and crystalline face of nanosized catalysts

Demonstrated of ability to monitor "single turnover events" (single catalytic events)

Nanostructured catalysts introduced in production after 2000 represent $30-40 \%$ worldwide of all catalysts
Store light with millisecond storage times or longer: although "slowed" light has been demonstrated, truly stored light has not; this could come about through extremely high-Q, lowloss resonant structures

Use plasmonic enhanced-emission and detection to achieve controlled absorption and emission of light from single molecules

Create "superlenses" for ultra-high-resolution imaging and "cloaking" at multiple wavelengths (a change of paradigm in optics)

Achieve "thresholdless" lasers, where efficiency of energy transfer is so great that lasing can be initiated with minuscule power input to achieve exceptionally high power gains

Achieve a complete snapshot of a multistep catalytic process

Ensure robustness and stability of nano-sized catalysts

Overall goal: precise control of composition and structure of catalysts over length scales spanning $1 \mathrm{~nm}$ to $1 \mu \mathrm{m}$, allowing the efficient control of reaction pathways

New nanostructured catalysts will cover at least $50 \%$ of the market worldwide by 2020

\section{Emerging uses of nanomaterials}

Establishment of synthesis and separation strategies for producing monodisperse nanomaterials, e.g., chiralityseparated carbon nanotubes

Realization of bulk nanocomposites/coatings with predictable and unique properties based on monodisperse nanoscale building blocks (e.g., transparent conductors based on carbon nanotubes and graphene)

Evolution from microcrystalline to nanocrystalline metals, polymer micro-composites to nano-composites, and microscale to nanoscale particle coatings

Realization of lighter and higher conductivity materials for airplane, satellite, and spacecraft wiring

Realization of nanofluidic devices and systems

Introduction of cellulose wood fibers in nanocomposite materials
Develop a complete library of monodisperse nanomaterials at industrial-scale quantities

Realize hierarchical nanostructured materials with independent tunability of previously coupled properties, (e.g., decoupling optical and electrical properties for photovoltaics, decoupling electrical and thermal properties for thermoelectrics)

Nanocomposite coatings with improved mechanical, thermal, chemical, electrical, magnetic, and optical properties compared to current state of the art

Realize nanocomposites for structural components, thus enabling $40 \%$ weight reduction in airplane designs with better overall performance

Achieve scalable nanofluidic systems for processing in biotechnology, pharmaceuticals, and chemical engineering

Mass use in nanotechnology of renewable and earth-abundant raw materials 
Appendix 1 continued

Main achievements/discoveries since 2000

Fundamental goals/"Holy Grails" to attain

and barriers to overcome by 2020

\section{Research facility infrastructure}

Rapid expansion of interdisciplinary nanoscience user centers, including large-scale facilities, as engines for interdisciplinary science and engineering discovery

Establishment of over 150 interdisciplinary research centers and user facilities in the United States and many others worldwide, providing broad access to fabrication and characterization facilities

Creation of the Nanoscale Computation Network in 2002, redesign of the National Nanotechnology Infrastructure Network in 2003, and establishment of the Network for Informal Science Education in 2004, providing more democratic and global access to nanoscale science and engineering knowledge and tools
Expand the breadth of interdisciplinary center capabilities and extend the geographical distribution for more widespread access

Create open access centers and hubs as test beds for development and maturation of innovative nano-enabled device and system concepts

Achieve widespread use of net-based remote control of instrumentation and local technician support at research facilities to enable reduced travel requirements and increased access by students

\section{Education infrastructure}

Nanotechnology has begun to foster interdisciplinary perspectives on science and engineering at all levels of STEM education

NanoHub, NISE, and the NACK Nano Education Portal of the NCLT are providing web-based access to nanoscale science and engineering resources

Publication of more than 50 textbooks for college-level nanoscale science and engineering courses leading to degrees with nanoscale science and engineering minors (and some majors) and/or certificates

Nanotechnology has emerged as a topic of interest on websites, in exhibits, and in educational programs at science museums around the world and across the country, including at Walt Disney World's Epcot Center
Embed nanoscale science and engineering education in internationally benchmarked standards and curriculum at all levels of education, but especially in the $\mathrm{K}-12$ developmental progression

Establish a network of regional hub sites - the

"Nanotechnology Education Hub Network"-with a sustainable infrastructure

Migrate education for the nanoscale from a supplement to traditional disciplines into its own specialties, e.g., nanoeducation organizations, degrees, and professional disciplines

Incorporate nanoscale science and engineering into all levels of STEM education, and "reverse the pyramid of leaning" by earlier inclusion of unifying concepts about nature, biology, information and cognition

\section{Governance}

Establishment of specific methods for governance of nanotechnology: bottom-up multi-agency governance approach, multi stakeholder assessment, scenario development

Establishment of an international community of professionals and organizations, including for nanotechnology EHS and ELSI

Developments in nomenclature, patents, standards, and standard materials

Creation of investment programs across disciplines, areas of application, and funding agencies
Emplace new principles and organizations for risk governance of new generations of nanotechnology products and processes with increased complexity, dynamics, biology contents, and uncertainty

Prepare the knowledge, people, and regulatory capacity to address mass use of nanotechnology by 2020

Create internationally recognized reference materials, terminology, materials certification, and measurement standards for nanomaterials

Institutionalize funding programs for nanotechnology research, education, manufacturing, and nanotechnology EHS and ELSI, applicable from passive nanostructures to active nanosystems 
Appendix 1 continued

\begin{tabular}{|c|c|}
\hline Main achievements/discoveries since 2000 & $\begin{array}{l}\text { Fundamental goals/“Holy Grails" to attain } \\
\text { and barriers to overcome by } 2020\end{array}$ \\
\hline $\begin{array}{l}\text { Establishment of a "Nanotechnology in Society" network with } \\
\text { funding comparable to that of hard science projects }\end{array}$ & $\begin{array}{l}\text { Institutionalize earlier integration of societal implications } \\
\text { programs with hard-science programs, and of research, } \\
\text { production and regulation organizations }\end{array}$ \\
\hline $\begin{array}{l}\text { Establishment of nanoinformatics as a new field for } \\
\text { communication, design, manufacturing, and medicine in } \\
\text { nanotechnology }\end{array}$ & $\begin{array}{l}\text { Develop a national and international network for } \\
\text { nanoinformatics }\end{array}$ \\
\hline $\begin{array}{l}\text { Development of a "scenarios approach" for nanotechnology } \\
\text { foresight and governance }\end{array}$ & $\begin{array}{l}\text { Increase international funding mechanisms for nanotechnology } \\
\text { areas of common use and benefit as nanotechnology becomes } \\
\text { a progressively more socio-economic undertaking }\end{array}$ \\
\hline $\begin{array}{l}\text { Initiation of funding programs for industry-inspired } \\
\text { fundamental research with industry sectors using } \\
\text { Collaborative Boards for Advancing Nanotechnology } \\
\text { (CBAN) }\end{array}$ & $\begin{array}{l}\text { Integrate discovery and innovation programs into public- } \\
\text { private-partnership platforms where academics, industry, } \\
\text { economists, and regulators are involved in all stages of the } \\
\text { innovation process }\end{array}$ \\
\hline
\end{tabular}

